Revista de MATEMÁtica: TeORÍA y APliCACIONEs 2015 22(2) : 325-339

CIMPA - UCR ISSN: 1409-2433 (PRINT), 2215-3373 (ONLINE)

\title{
OPTIMIZACIÓN DE LA OFERTA DE CURSOS EN INSTITUCIONES EDUCATIVAS
}

\section{OPTIMIZATION OF COURSES OFFER IN EDUCATIONAL INSTITUTIONS}

\author{
RAFAEL LÓPEZ-BRACHO* \\ ANGÉliCA ILIANA GRANADOS OCHOA ${ }^{\dagger}$ \\ Mirelle Hernández Fragoso
}

Received: 14/Mar/2012; Revised: 7/Apr/2015;

Accepted: 7/May/2015

* Sistemas, Universidad Autónoma Metropolitana- Azcapotzalco. México D.F., México. EMail: rbl@correo.azc.uam.mx

${ }^{\dagger}$ Misma dirección que/Same address as: R. López-Bracho E-Mail: angelica.grana2@outlook.com

${ }^{\ddagger}$ Misma dirección que/Same address as: R. López-Bracho E-Mail: mhdzf020986@gmail.com 


\title{
Resumen
}

En el problema de programación de horarios de cursos, generalmente se asume que la asignación de asignaturas a los periodos lectivos es un dato de entrada del problema, que es definido por los responsables académicos. En este trabajo es presentada una metodología, que hace uso de programas lineales con variables enteras $0-1$, para establecer la asignación de cursos a periodos lectivos, el cual toma en cuenta el grado de dificultad de cada asignatura y los requisitos académicos de éstas, con el objetivo de minimizar las diferencias de grado de dificultad entre los diferentes periodos académicos.

Palabras clave: programación entera; horarios; asignación; optimización.

\begin{abstract}
In the problem of scheduling of courses, it is generally assumed that the allocation of subjects to teaching periods is an input of the problem, which is defined by the academic authorities. This paper presents a methodology that makes use of linear programs with $0-1$ integer variables, to establish the assignment of subjects to teaching periods, which takes into account the degree of difficulty of each subject and academic requirements of these, with the objective of minimizing the differences in degree of difficulty between the various academic periods.
\end{abstract}

Keywords: integer programming; timetabling; allocation; optimization.

Mathematics Subject Classification: 90C10, 90C90.

\section{Presentación}

En este trabajo se presenta una metodología para definir la oferta de cursos en cada periodo lectivo de una institución educativa, de manera que con base en los recursos disponibles se pueda optimizar la atención a la demanda que presentan los alumnos, respetando la flexibilidad curricular de los planes de estudio. La metodología hace uso de dos modelos de programación entera para establecer las asignaturas que se deben abrir en los diferentes periodos lectivos.

Los modelos tienen como objetivo establecer un equilibrio en el nivel de dificultad de la carga académica propuesta a los alumnos en cada periodo lectivo, que tenga como consecuencia mejorar la eficiencia terminal de los planes de estudio, respetando la disponibilidad de profesores y aulas.

El primer modelo es aplicable a conjuntos de asignaturas obligatorias en el plan de estudios y resulta en una selección de asignaturas para cada periodo lectivo, de manera que se minimiza la diferencia entre los grados de dificultad 
de los periodos. El segundo modelo es aplicable a conjuntos de asignaturas optativas en el plan de estudios y resulta en la selección de las optativas que se ofrecerán en cada periodo lectivo de manera que maximice la atención a la demanda de los alumnos. Los modelos se probaron usando datos estadísticos de una universidad, en la cual el alumno tiene la libertad de hacer su horario, con las asignaturas que él libremente elige de la oferta que presenta la institución educativa. Se muestran los resultados obtenidos.

\section{Necesidad de definir la oferta por periodo lectivo}

Tradicionalmente, en los problemas de programación de horarios de cursos, la oferta de asignaturas y el número de grupos de cada una se consideran valores conocidos, y están en la misma categoría que la disponibilidad de aulas, profesores y demanda de los alumnos, es decir son los datos de entrada del problema. La asignación de profesores, aulas y horarios a los cursos propuestos es un problema que se ha modelado mediante instancias del problema de asignación multidimensional y para el cual existen muchos estudios y propuestas de heurísticos para abordarlos [1], [3], [5], [8]. En cambio, la definición de la oferta de cursos a programar por periodo lectivo es un problema que no ha sido suficientemente abordado como problema de optimización.

\subsection{Flexibilización de los planes de estudio}

Recientemente, al flexibilizar el currículo de las universidades para permitir que cada alumno defina un plan de estudios personal, a partir de un mayor número de asignaturas optativas y menos restricciones de precedencia (seriación) entre materias, ha cambiado la forma en que se presenta la demanda de los cursos que se imparten, surgiendo la necesidad de programar los cursos con mayor frecuencia de la que en realidad se requeriría. Esta situación provoca que sea más complejo para los coordinadores de estudio decidir que asignaturas y cantidad de grupos abrir en cada periodo lectivo. Además, si bien la flexibilidad curricular otorga al alumno la libertad de hacer el horario con las asignaturas que él libremente elige, en ocasiones puede tener desventajas, pues sin un apoyo para tomar la decisión de que asignaturas conviene cursar en un periodo lectivo, de manera que la carga académica sea balanceada, puede ocurrirle que sólo avance en ciertas áreas de conocimiento o que en ciertos periodos lleve materias que no necesitan tanta dedicación o por el contrario, que inscriba asignaturas en las que la dedicación tiene que ser mayor, ocasionando que el alumno repruebe, obtenga 
una baja calificación o tome la decisión de dar de baja la inscripción a la materia, convirtiéndolo en un alumno irregular.

Un caso particular de esta situación se presenta en la Unidad Azcapotzalco de la Universidad Autónoma Metropolitana (UAM-A), de la Ciudad de México, en la que, en sus programas de ingeniería cada alumno define su propio avance curricular, respetando el número máximo de créditos que puede llevar por trimestre y las restricciones de seriación entre asignaturas. Los estudios se realizan en doce trimestres, tres por año, durante cuatro años y los alumnos van seleccionando en cada trimestre, a partir del segundo año, las asignaturas que van a cursar de la oferta programada. Aunque el sistema siempre ha sido flexible, al aumentar recientemente el número de optativas y disminuir las restricciones de seriación entre asignaturas, ha aumentado para los coordinadores de estudios la dificultad para definir en cada trimestre, las asignaturas que se van a programar y el número de grupos de cada una de ellas.

Ante el límite en la disponibilidad de profesores y aulas, no se puede abrir grupos de todas las asignaturas en cada trimestre, por lo que una solución es repartir la oferta de cursos en los tres trimestres de cada ciclo anual y hacer una propuesta de avance escolar de asignaturas obligatorias para los alumnos regulares, mientras que para las asignaturas optativas hacer la selección con base en la atención de la demanda presentada por una muestra de alumnos.

\subsection{Problema de programación de cursos}

A partir de la problemática antes referida, se propone un problema de programación entera 0-1 con el que se obtenga para cada curso el trimestre en el que deberá ser programado, con el número de grupos requerido para atender la demanda anual, de manera que se respeten las restricciones de seriación y de número máximo de créditos que puede un alumno cursar por trimestre. Para este problema se presentan dos variantes, una para las asignaturas obligatorias y otra para las asignaturas optativas.

En el problema de programación de cursos de asignaturas obligatorias, se toma como objetivo lograr un equilibrio en la dificultad de los trimestres, es decir que no haya mucha diferencia en la dificultad acumulada, de las asignaturas de cada par de trimestres. Desde luego se requiere una medida de la dificultad.

En el problema de programación de cursos optativos, se toma como objetivo maximizar la satisfacción de la demanda atendida, de las solicitudes de optativas presentadas por una muestra de alumnos.

Los dos modelos se presentan detalladamente en la sección siguiente. 


\section{Modelos propuestos para la selección de asignaturas a impartir}

Los modelos que se presentan atienden a la necesidad de proponer una oferta de asignaturas adecuada a la atención de dos necesidades específicas, según el tipo de asignaturas que se estén cursando. En el caso de asignaturas obligatorias, las cuales pueden ser comunes a varios planes de estudio, se propone un modelo que busca definir las asignaturas que se deben cursar en cada periodo lectivo, de manera que el grado de dificultad acumulado de las asignaturas sea similar entre un periodo y otro.

En el caso de asignaturas optativas, las cuales es común se cursen en la parte final de los estudios, se propone un modelo que busca establecer, para cada generación, las asignaturas que se van a ofrecer en los periodos lectivos correspondientes, de manera que se atienda en totalidad la demanda presentada por el mayor número de alumnos posible.

\subsection{Modelo de programación entera para definir la programación de asignaturas obligatorias}

El modelo propuesto se basa en tres aspectos fundamentales: La seriación de las asignaturas, el grado de dificultad de cada asignatura y el número mínimo y máximo de créditos que se pueden llevar en un periodo lectivo.

\subsubsection{Seriación de las asignaturas}

A partir de la construcción de un diagrama de seriación de las asignaturas, se identifican las diferentes secuencias de materias, denominando cadena a cada secuencia maximal, de manera que una asignatura puede estar presente en varias cadenas y una asignatura no seriada con ninguna otra constituye una cadena individual.

\subsubsection{Grado de dificultad}

El grado de dificultad de cada asignatura se calcula a partir de datos estadísticos acumulados de varios periodos lectivos de impartición de la asignatura, utilizando tres criterios: Número de alumnos inscritos, número de alumnos que dieron de baja la asignatura y número de alumnos que reprobaron. Con estos datos se obtiene el porcentaje total de no aprobación como sigue:

$\%$ de no aprobación $=\left(\frac{\text { total de alumnos reprobados+total de alumnos dados de baja }}{\text { total de alumnos inscritos }}\right) \times 100$. 
El recíproco del porcentaje de no aprobación es el grado de dificultad de cada asignatura, éste se interpreta como el número promedio de intentos u oportunidades que un alumno necesita para aprobar la materia, la fórmula correspondiente es:

$$
\text { grado de dificultad }=\left(\frac{1}{\% \text { de no aprobación de la asignatura }}\right) .
$$

Finalmente, con base en este dato se puede calcular el grado de dificultad por crédito de la asignatura, utilizando la siguiente fórmula:

grado de dificultad por crédito de la asignatura $=\left(\frac{\text { grado de dificultad de la asignatura }}{\text { número de créditos de la asignatura }}\right)$.

\subsubsection{Mínimo y máximo de créditos por periodo lectivo}

El valor mínimo y máximo del número de créditos que se pueden llevar en un periodo lectivo está definido en cada plan de estudios.

\subsection{Modelo}

A partir de esta información se propone el modelo siguiente:

\section{- Índices y variables:}

- Índices: $i \in I$, conjunto de asignaturas; $j \in J$, conjunto de períodos lectivos.

- Variables de decisión: $x_{i j}$ que asocia a la asignatura $i$ con el periodo lectivo $j$, donde

$$
x_{i j}=\left\{\begin{array}{l}
1 \text { si la asignatura } i \text { se impartirá en el periodo lectivo } j \\
0 \text { si no. }
\end{array}\right.
$$

- Datos y variables de exceso y holgura del modelo:

- $C_{i}$ : créditos de la asignatura $i$.

- $d_{i}$ : grado de dificultad por crédito de la asignatura $i$.

- $g_{j_{1}}, g_{j_{2}}$ : déficit y exceso de dificultad acumulada con respecto al grado de dificultad promedio, en el periodo $j$, respectivamente. 
- Función objetivo: Minimiza la suma del grado de dificultad en exceso o déficit respecto al esperado, para cada periodo lectivo considerado en el plan de estudios

$$
\min \mathrm{z}=\sum_{j=1}^{n}\left(g_{j_{1}}+g_{j_{2}}\right)
$$

donde $n$ es el número de periodos considerados en la planeación de las asignaturas.

\section{- Restricciones:}

- Restricciones de créditos,

$$
\begin{aligned}
& \sum_{i=1}^{m} C_{i} x_{i j} \leq C M P \\
& \sum_{i=1}^{m} C_{i} x_{i j} \geq C M N P
\end{aligned}
$$

donde $m$ es el número total de asignaturas, $C M P$ es el número máximo de créditos permitidos por alumno por periodo y $C M N P$ es el número mínimo de créditos permitidos por alumno por periodo. Se aplica para cada periodo lectivo.

- Restricción del grado de dificultad acumulado por las asignaturas del periodo,

$$
\sum_{i=1}^{m}\left(C_{i}\left(d_{i}-\bar{g}\right) x_{i j}\right)+g_{j_{1}}-g_{j_{2}}=0
$$

donde $\bar{g}$ es la dificultad por crédito promedio del total de las asignaturas. Se aplica para cada periodo del plan de estudios.

- Restricciones de cadena,

$$
X_{i_{k}, k}+X_{i_{k}, k+1}+\ldots+X_{i_{k}, n-p+k}=1, \quad i_{k}=1,2, \ldots, m
$$

donde $n$ es el número de periodos considerados, $k$ es la posición de la asignatura $i_{k}$ en la cadena, $1 \leq k \leq p$ y $p$ es el número de asignaturas encadenadas. Se aplica para todas las cadenas a las que pertenezca cada variable.

- Restricciones de seriación,

$$
X_{i_{k}, j} \leq X_{i_{k+1}, j+1}+X_{i_{k+1}, j+2}+\cdots+X_{i_{k+1}, n-p+k+1}
$$

Se aplica a cada par de asignaturas en secuencia de seriación en alguna cadena. 
- Programa lineal mixto con enteros $0-1$ :

$$
\min z=\sum_{j=1}^{n}\left(g_{j_{1}}+g_{j_{2}}\right)
$$

sujeto a

$$
\begin{array}{r}
\sum_{i=1}^{m} C_{i} x_{i j} \leq C M P, j=1,2, \ldots, n \\
\sum_{i=1}^{m} C_{i} x_{i j} \geq C M N P, j=1,2, \ldots, n \\
\sum_{i=1}^{m}\left(C_{i}\left(d_{i}-\bar{g}\right) x_{i j}\right)+g_{j_{1}}-g_{j_{2}}=0, j=1,2, \ldots, n \\
X_{i_{k}, k}+X_{i_{k}, k+1}+\ldots+X_{i_{k}, n-p+k}=1, i_{k}=1,2, \ldots, m \\
X_{i_{k}, j \leq X_{i_{k+1}, j+1}+X_{i_{k+1}, j+2}+\ldots+X_{i_{k+1}, n-p+k+1}, i_{k}}=1,2, \ldots, m \\
X_{i j}=0,1 ; \quad i=1,2, \ldots, m ; j=1,2, \ldots, n \\
g_{j_{1}}, g_{j_{2}} \geq 0 ; j=1,2, \ldots, n .
\end{array}
$$

\subsection{Modelo de programación entera para definir la programación de asignaturas optativas}

Se consideran ahora planes de estudio que ofrecen la posibilidad de cursar asignaturas optativas. Se desea seleccionar las asignaturas que se van a programar en cada periodo lectivo, de manera que se pueda atender la demanda de inscripción solicitada por los alumnos. Para un número de asignaturas relativamente grande, se vuelve muy difícil hacer la programación de manera que se ofrezcan en cada año lectivo todas las materias que sean solicitadas por los alumnos, pero haciéndolo de forma óptima, esto es: Se deben ofrecer las asignaturas en un solo periodo lectivo si tienen poca demanda y en más de un periodo en el año o programando más grupos si tienen una gran demanda.

Para el modelo propuesto, se establece que la demanda se definirá por medio de una consulta a un subconjunto de los alumnos que estarán concluyendo sus estudios en el año lectivo a programar, y el modelo buscará maximizar la atención a la demanda de asignaturas optativas de este grupo de alumnos, de manera que la oferta de cursos les permita llevarlos en los diferentes periodos lectivos del año en forma equilibrada, esto es: sin que sea excesivo el número de créditos de las asignaturas a cursar en algún periodo. 
Si $m$ es el número de asignaturas optativas del programa, $n$ el número de alumnos a consultar y $r$ el número de asignaturas optativas que deben cursar, el número de selecciones de optativas diferentes asciende a $\left(\begin{array}{c}m \\ r\end{array}\right)$, de las cuales se van a tomar $n$ combinaciones. No tiene que ser muy grande $n$ para que se vuelva irresoluble atender todas las demandas en un número pequeño de periodos lectivos. Ante esta situación, se propone consultar únicamente a un número pequeño de alumnos regulares, quienes habrán demostrado con anterioridad compromiso e interés en terminar los cursos elegidos.

Con base en estas consideraciones se establece el modelo siguiente:

$$
\max Z=\sum_{i=1}^{m} \sum_{j=1}^{2 p} a_{i} x_{i j}-\sum_{k=1}^{n} \sum_{t=1}^{p}\left(v_{k t}+w_{k t}\right)
$$

sujeto a

$$
\begin{gathered}
\sum_{j=1}^{2 p} x_{i j} \leq 1, \quad i=1,2, \ldots, m \\
\sum_{i \in A_{k}}\left(x_{i, 2 t-1}+x_{i, 2 t}\right)+v_{k t}-w_{k t}=r_{t}, \quad k=1,2, \ldots, n ; t=1, \ldots, p \\
i j \in\{0,1\} ; \quad w_{k t} \in \mathbb{Z},
\end{gathered}
$$

donde

$x_{i j}=\left\{\begin{array}{l}1 \text { si un grupo se asigna en el periodo } j, \\ 0 \text { si no } \\ i=1, \ldots, m ; j=1, \ldots, p\end{array}\right.$

$m$ es el número de asignaturas optativas del plan de estudios,

$p$ es el número de periodos lectivos,

$a_{i}$ es el número de alumnos consultados que seleccionaron la asignatura optativa i para ser cursada,

$A_{k}$ es el conjunto de asignaturas optativas seleccionadas por el alumno $k$,

$r_{t}$ es el número de asignaturas optativas que se sugiere cursar en el periodo $t$, $v_{k t} \mathrm{y} w_{k t}$ son el número de asignaturas optativas faltantes y en exceso, respectivamente, que podrá cursar el alumno $k$ en el trimestre $t$, con base en la programación obtenida y el número de optativas sugeridas para el periodo lectivo.

\section{Ejemplo de aplicación}

El modelo educativo de las licenciaturas en ingeniería de UAM-A da al alumno la libertad de hacer el horario con las asignaturas que él libremente elige. 
Es un modelo muy flexible que tiene las características planteadas para los modelos. Las asignaturas de ingeniería, se clasifican en cada plan de estudio en tres niveles, que corresponden a la aportación de conocimientos que brindan a los alumnos, el avance en el plan de estudios y la importancia en el logro de objetivos de la licenciatura. Los dos primeros niveles corresponden a asignaturas obligatorias y el tercero contiene asignaturas tanto obligatorias como optativas. Los niveles de asignaturas son los siguientes: Tronco básico general, Tronco básico profesional y Área de concentración, respectivamente.

En el proyecto terminal Optimización de la Oferta de Cursos en la División de Ciencias Básicas e Ingeniería [4] se reporta el ejercicio de aplicación del modelo propuesto para asignaturas obligatorias, el cual se aplicó al conjunto de materias del Tronco básico profesional y al conjunto de materias obligatorias del Área de concentración de la licenciatura en Ingeniería Industrial, mientras que el modelo para asignaturas optativas únicamente se formuló para el conjunto de materias optativas del Área de concentración de la misma licenciatura. Los resultados obtenidos se presentan a continuación.

\subsection{Implementación del modelo para asignaturas obligatorias}

Al observar que para atender el tronco básico profesional de la licenciatura en Ingeniería Industrial, la Universidad debía programar 34 asignaturas, como primera medida de la propuesta se decidió agrupar las materias teóricas con su taller o laboratorio, quedando así, para el ejercicio de planeación, materias de 12 ó 15 créditos teórico-prácticas y materias de nueve o seis créditos que sólo son teóricas, reduciendo el modelo a 25 asignaturas. Para estimar el grado de dificultad de cada asignatura, se usaron datos estadísticos de 16 trimestres, del 05P al 10P, obtenidos en la dirección de resultados estadísticos de registro escolar de la Unidad Azcapotzalco [2]. Los datos consultados corresponden, para cada grupo de cada asignatura, al número total de alumnos inscritos, el número de alumnos que solicitaron baja de la inscripción y el número de alumnos aprobados. Considerando la gran variabilidad que puede haber entre un grupo y otro, debido a la impartición de los cursos por distintos profesores, se optó por usar medias truncadas en la obtención de los índices requeridos para generar el grado de dificultad de cada asignatura. Para este estudio no había la intención de hacer pruebas estadísticas formales, por lo que se optó por usar herramientas estadísticas de las técnicas de pronósticos [9]. La lista de asignaturas, junto con su grado de dificultad se muestra en la tabla 1. Por otra parte, el plan de estudios de la licenciatura en Ingeniería Industrial establece que los alumnos de tiempo completo deben inscribir entre 33 y 45 créditos por trimestre, por lo que las asignaturas del tronco básico profesional deberán cursarse en seis trimestres, trimestres 4 al 9 , 


\begin{tabular}{|c|c|c|c|c|c|}
\hline Número & Asignatura & Requisitos & Créditos & $g_{i}$ & $d_{i}=g_{i} /$ créditos \\
\hline 1 & $\begin{array}{l}\text { Circuitos Eléctricos } \\
\text { Laboratorio de Circuitos Eléctricos }\end{array}$ & - & 12 & 3.6917 & 0.3076 \\
\hline 2 & $\begin{array}{l}\text { Introducción a los Materiales } \\
\text { Laboratorio de Introducción a los Materiales }\end{array}$ & - & 9 & 2.9374 & 0.3264 \\
\hline 3 & Economía Mexicana & - & 9 & 1.4721 & 0.1636 \\
\hline 4 & Probabilidad y Estadística & - & 9 & 2.3254 & 0.2584 \\
\hline 5 & Introducción a las Estructuras I & - & 9 & 2.7749 & 0.3083 \\
\hline 6 & $\begin{array}{l}\text { Laboratorio de Ingeniería Eléctrica } \\
\text { Ingeniería Eléctrica }\end{array}$ & - & 15 & 2.6639 & 0.1776 \\
\hline 7 & $\begin{array}{l}\text { Introducción a Operaciones Unitarias } \\
\text { Laboratorio de Operaciones Unitarias }\end{array}$ & 8,9 & 12 & 3.1881 & 0.2657 \\
\hline 8 & $\begin{array}{l}\text { Fisicoquímica de los Materiales } \\
\text { Laboratorio de Fisicoquímica }\end{array}$ & 8,9 & 12 & 3.1881 & 0.2657 \\
\hline 9 & Termodinámica Aplicada I & - & 9 & 2.9313 & 0.3257 \\
\hline 10 & Mediciones en Ingeniería I & 4 & 6 & 1.1700 & 0.1950 \\
\hline 11 & Investigación de Operaciones I & - & 9 & 2.1612 & 0.2401 \\
\hline 12 & Investigación de Operaciones II & 4,11 & 9 & 1.3635 & 0.1515 \\
\hline 13 & Labor de Análisis y Simulación de Sistemas & - & 6 & 1.4919 & 0.2487 \\
\hline 14 & Análisis de Decisiones I & 75 créditos & 9 & 1.8230 & 0.2026 \\
\hline 15 & Control de Calidad y Confiabilidad & - & 9 & 1.1981 & 0.1331 \\
\hline 16 & Metrología para Manufacturas & - & 6 & 1.7037 & 0.2840 \\
\hline 17 & México, Política y Sociedad & - & 9 & 1.3322 & 0.1480 \\
\hline 18 & $\begin{array}{l}\text { Diodos y Amplficadores Operacionales } \\
\text { Labo de Diodos y Amplif Operacionales }\end{array}$ & 1 & 12 & 2.7487 & 0.2291 \\
\hline 19 & $\begin{array}{l}\text { Instrumentación Industrial } \\
\text { Laboratorio de Instrumentación Industrial }\end{array}$ & 18 & 9 & 2.2798 & 0.2533 \\
\hline 20 & $\begin{array}{l}\text { Procesos de Manufactura I } \\
\text { Taller de Procesos de Manufactura I }\end{array}$ & 2 & 12 & 2.4390 & 0.2033 \\
\hline 21 & $\begin{array}{l}\text { Procesos de Manufactura II } \\
\text { Taller de Procesos de Manufactura II }\end{array}$ & 20 & 12 & 2.2868 & 0.1906 \\
\hline 22 & Economía Industrial & 3 & 9 & 1.3311 & 0.1479 \\
\hline 23 & Organización Industrial & 22 & 9 & 1.6304 & 0.1812 \\
\hline 24 & Estadística Aplicada I & 4 & 9 & 1.4895 & 0.1655 \\
\hline \multirow[t]{2}{*}{25} & Introducción a las Estructuras II & 5 & 9 & 1.9880 & 0.2209 \\
\hline & & & 240 & 54.0159 & 0.22506625 \\
\hline
\end{tabular}

Tabla 1: Relación de asignaturas teóricas de tronco básico profesional.

mientras que las asignaturas obligatorias de área de concentración juntos con las asignaturas optativas en tres trimestres, trimestres 10 al 12.

Al incorporar las restricciones de cadena y de seriación se obtuvo para las asignaturas de tronco básico profesional un programa lineal entero mixto con 25 variables 0-1, 2 variables continuas y 81 restricciones, el cual se resolvió con una aplicación del paquete LINGO, cuyo resultado permitió elaborar la propuesta de programación de las asignaturas correspondiente, mostrada en la Tabla 2.

\subsection{Formulación del modelo para asignaturas optativas}

La formulación del modelo para asignaturas optativas requirió de un examen más cuidadoso. El plan de estudios de Ingeniería Industrial proponía, en el momento 


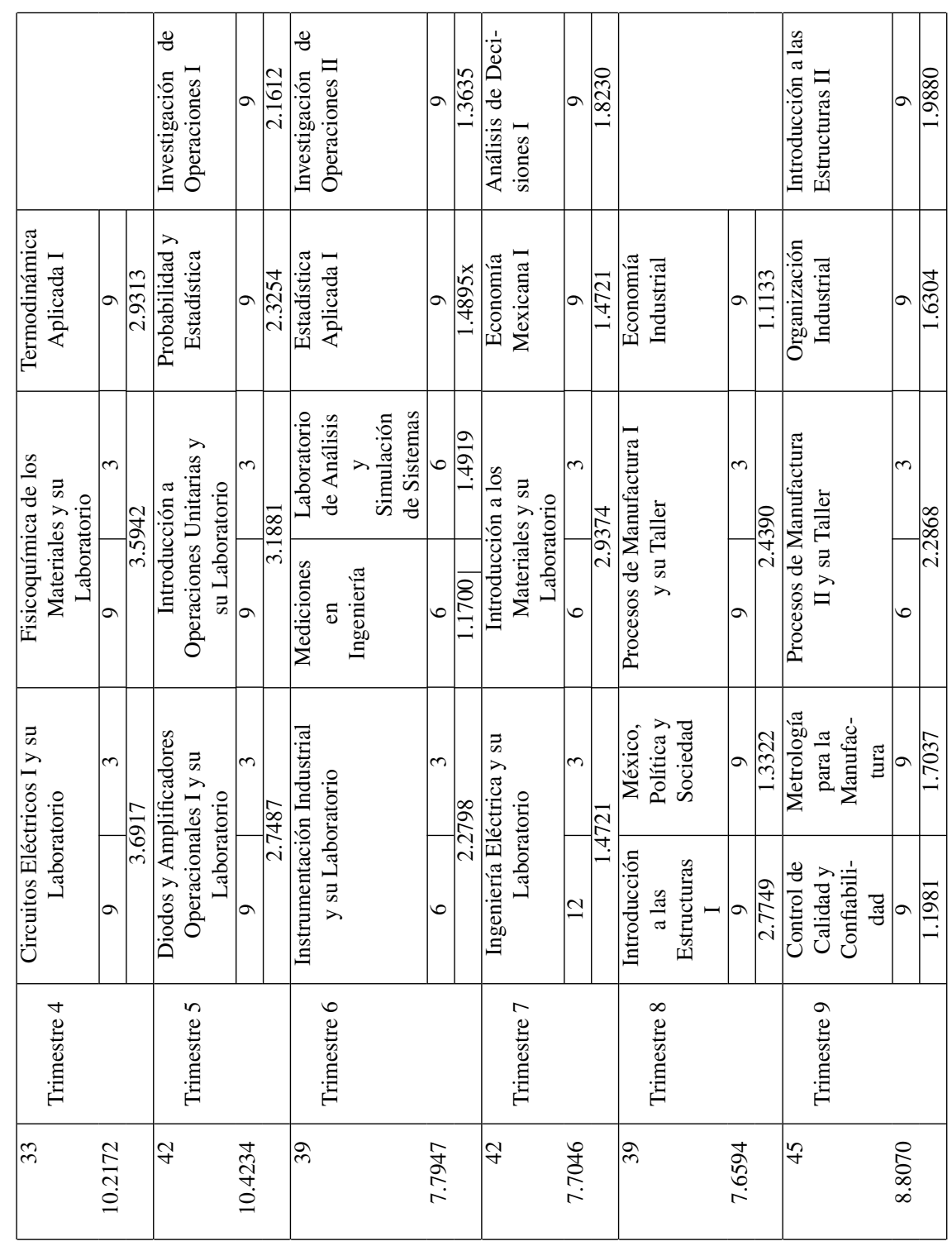

Tabla 2: Resultados del modelo del tronco básico profesional, $\bar{Z}=12.341495$. 
del estudio, que los alumnos cursaran al menos siete asignaturas optativas a seleccionar de una oferta de 33 asignaturas de las cuales siete eran obligatorias del Tronco Básico Profesional de otras ingenierías, por lo que quedarían 26 asignaturas a considerar. Además históricamente ha existido una gran variabilidad en el número de estudiantes que han cursado cada asignatura.

La oferta de asignaturas optativas ha dependido mucho de la costumbre y de la formación e interés profesional de los profesores, y en un menor grado de la demanda manifiesta de los alumnos. En una consulta, no una prueba formal estadística, realizada a egresados de la licenciatura sobre las asignaturas optativas que tomaron, más del $40 \%$ manifestó que hubo optativas que les hubiera gustado cursar pero nunca se programaron o fueron programadas en un horario inadecuado, y un porcentaje similar aseveró que tomaron las asignaturas que había programadas cuando así lo requirieron sin manifestar preferencia por otras. Los resultados de la consulta están reportados en [4].

Por otra parte, al momento del estudio, estadísticas de la Universidad mostraban [6] que en los últimos seis años se había obtenido un promedio, con poca varianza, de 88 alumnos egresados cada año de la licenciatura en Ingeniería Industrial, de los cuales aproximadamente el 15\% (13 alumnos) estaban concluyendo con un retraso menor a un año, del tiempo normal (cuatro años) establecido en el Plan de Estudios.

Los resultados arriba expuestos sugieren lo siguiente: Se tienen $\left(\begin{array}{c}26 \\ 7\end{array}\right)$ posibilidades de elección de siete optativas propias del plan, son 88 egresados en promedio, los que tienen que integrar su conjunto de optativas cada año, siendo 13 aproximadamente los que estarían concluyendo sin un retraso mayor, esto es, habían mantenido un avance regular en sus estudios. Se podría entonces hacer una consulta cada año a los alumnos regulares para que sean éstos quienes definan, con sus solicitudes, las asignaturas optativas que se deberían programar en los tres trimestres del último año de estudios. Si eligieran asignaturas de mucha demanda, lo más probable es que se ofrecerían en todos los trimestres, en cambio cuando solicitaran asignaturas de pequeña demanda, se programaría un solo curso para cada una de ellas. Esto se haría, resolviendo el modelo para asignaturas optativas, de manera que los alumnos consultados tuvieran horarios de carga similar en los tres trimestres en la medida de lo posible. Estos alumnos además, garantizarían que los cursos de poca demanda no se cancelaran por baja de los alumnos. El espíritu del modelo propuesto es atender las ideas arriba planteadas y sirve de reconocimiento a los alumnos regulares, a quienes se les abrirían cursos aunque tengan una demanda pequeña. El modelo, ya está explicado en la sección 3.2 y aquí únicamente se establece como se integraría el conjunto de alumnos decisores de optativas. 


\section{Resultados y conclusiones}

La metodología propuesta para elaborar la planeación anual de asignaturas a ofrecer a los alumnos de una licenciatura, probada en la licenciatura en Ingeniería Industrial de UAM Azcapotzalco, es aplicable a cualquier otra licenciatura que cuente con los datos históricos de aprobación, reprobación y bajas de las asignaturas impartidas en la propia institución.

Se pensó en una planeación anual, para que el alumno pueda saber con anticipación que asignaturas se impartirán en el año y pueda planear mejor su carga académica. Llevar una planeación anual sólo compromete a la institución a buscar siempre la optimización de sus recursos, a buscar y desarrollar herramientas que le permitan llevar sus planes a cabo o incluso cambiarlos y mejorarlos.

En este trabajo se abordó también la problemática que aqueja a muchos alumnos, de cómo decidir que asignatura cursar, ¿Cómo elegir?, las posibilidades son tan variadas que muchas veces hasta que se está por concluir los estudios el alumno se da cuenta, con base en la experiencia, de la dificultad de cada asignatura. Se tomó esto en cuenta para elaborar la tabla 2, como una guía de las asignaturas que se pueden cursar en cada trimestre y que la institución se comprometería a ofrecer con un número suficiente de capacidad en los grupos, de manera que otorgaran a los alumnos la posibilidad de llevar un avance regular, manteniendo una carga balanceada en cuanto al grado de dificultad y variedad de áreas de conocimiento.

Para poder realizar una planeación anual de asignaturas no sólo es necesario saber que materias impartir en cada periodo lectivo, es fundamental conocer la demanda de cada asignatura para tener un buen resultado, para esto no se tuvo que implementar un algoritmo, sólo se aplicaron técnicas de pronósticos y estudios estadísticos.

Se puede decir que tablas similares a la tabla 2 se pueden construir para cada una de las licenciaturas, y con base en éstas realizar una propuesta anual. Además, al generar una planeación basada en el grado de dificultad de las asignaturas, se podría llegar a disminuir los índices de no aprobación y así también disminuir el tiempo que un alumno necesita para concluir sus estudios.

\section{Referencias}

[1] Burke, E. K.; Jackson, K.; Kingston, J.; Weare, R. (1997) "Automated university timetabling: The state of the art", The Computer Journal 40(9): $565-571$. 
[2] División de Ciencias Básicas e Ingeniería, Ingeniería Industrial, MarzoMayo 2011, en: http://www2.azc.uam.mx/siga/, consultado del 16/03/2011 al 15/05/2011.

[3] Ernst, A. T.; Jiang, H.; Krishnamoorthy, M; Sier, D. (2004) "Staff scheduling and rostering: A review of applications, methods and models", European Journal of Operational Research 153(1): 3-27.

[4] Granados Ochoa, A.I.; Hernández Fragoso, M. (2011) Optimización de la Oferta de Cursos en la División de Ciencias Básicas e Ingeniería. Proyecto terminal de Ingeniería Industrial, UAM-Azcapotzalco, Ciudad de México.

[5] Hernández R.; Miranda J.; Rey P. (2008) "Programación de horarios de clases y asignación de salas para la Facultad de Ingeniería de la Universidad Diego Portales mediante un enfoque de programación entera", Revista de Ingeniería de Sistemas 22: 121-141.

[6] Informe del Rector General (2010) Anuario estadístico 2010, en: http : / / www . uam.mx/transparencia/inforganos/anuarios/ anuario2010/anuario_estadistico_2010.pdf, consultado el 31/08/2011, 9:30 p.m.

[7] Johnson, K. (1980) Timetabling. Hutchinson Educ., Londres.

[8] Lara-Velázquez, P.; López-Bracho, R.; Ramírez-Rodríguez, J.; Yáñez, J. (2011) "A model for timetabling problems with period spread constraints", Journal of the Operational Research Society 62(1): 217-222.

[9] Makridakis, S.; Wheelwright, S.; Hyndman, R. (1998) Forecasting: Methods and Applications. John Wiley \& Sons, New York.

[10] Murty, K.G. (1995) Operations Research: Deterministic Optimization Models. Prentice Hall, Englewood Cliffs NJ.

[11] Nemhauser, G.; Wolsey L. (1999) Integer and Combinatorial Optimization. John Wiley \& Sons, New York.

[12] Schrage, L. (1997) Lingo User's Manual. Boyd \& Fraser, Danvers MA. 
